\title{
SOME OPEN PROBLEMS AND CONJECTURES ON SUBMANIFOLDS OF FINITE TYPE: RECENT DEVELOPMENT
}

\author{
BANG-YEN CHEN
}

\begin{abstract}
Submanifolds of finite type were introduced by the author during the late 1970s. The first results on this subject were collected in author's books [26, 29]. In 1991, a list of twelve open problems and three conjectures on finite type submanifolds was published in [40]. A detailed survey of the results, up to 1996, on this subject was given by the author in [48]. Recently, the study of finite type submanifolds, in particular, of biharmonic submanifolds, have received a growing attention with many progresses since the beginning of this century. In this article, we provide a detailed account of recent development on the problems and conjectures listed in [40].
\end{abstract}

\section{Introduction}

Algebraic geometry studies algebraic varieties which are defined locally as the common zero sets of polynomials. In algebraic geometry, one can define the degree of an algebraic variety by its algebraic structure. The concept of degree plays a fundamental role in algebraic geometry. On the other hand, according to Nash's imbedding theorem, every Riemannian manifold can be realized as a Riemannian submanifold in some Euclidean space with sufficiently high codimension. However, one lacks the notion of the degree for Riemannian submanifolds in Euclidean spaces.

Inspired by the above simple observation, the author introduced in the late 1970s the notions of "order" and "type" for submanifolds of Euclidean spaces and used them to introduce the notion of finite type submanifolds. Just like minimal submanifolds, submanifolds of finite type can be characterized by a spectral variational principle; namely, as critical points of directional deformations [66].

The family of submanifolds of finite type is huge, which contains many important families of submanifolds; including minimal submanifolds of Euclidean space, minimal submanifolds of hyperspheres, parallel submanifolds as well as all equivariantly immersed compact homogeneous submanifolds.

Received December 22, 2013, accepted January 15, 2014.

2010 Mathematics Subject Classification. Primary: 53-02, 53C40, 53C42; Secondary 58G25.

Key words and phrases. Finite type submanifold, biharmonic submanifold, biharmonic conjecture, Chen's conjecture, linearly independent immersion, orthogonal immersion. 
On one hand, the notion of finite type submanifolds provides a very natural way to apply spectral geometry to study submanifolds. On the other hand, one can also apply the theory of finite type submanifolds to investigate the spectral geometry of submanifolds. For instance, the author was able to obtain sharp estimates of the total mean curvature for compact submanifolds of Euclidean space via his theory of finite type submanifolds.

The first results on submanifolds of finite type were collected in [26, 29]. A list of twelve open problems and three conjectures on submanifolds of finite type was published in 1991 [40]. Furthermore, a detailed report of the progress on this theory, up to 1996, was presented in [48].

Recently, the study of finite type submanifolds, in particular, of biharmonic submanifolds, have received a growing attention with many progresses since the beginning of this century. In this article, we provide a detailed account of recent development on the problems and conjectures listed in [40].

\section{Preliminaries}

\subsection{Finite type submanifolds}

We recall some basic definitions, results and formulas (for more details see for instance $[26,52])$.

Let $x: M \rightarrow \mathbb{E}^{m}$ be an isometric immersion of a (connected) Riemannian manifold $M$ into the Euclidean $m$-space $\mathbb{E}^{m}$. Denote by $\Delta$ the Laplace operator of $M$. The immersion $x$ is said to be of finite type if the position vector field of $M$ in $\mathbb{E}^{m}$, also denoted by $x$, can be expressed as a finite sum of $\mathbb{E}^{m}$-valued eigenfunctions of the Laplace operator, i.e., if $x$ can be expressed as

$$
x=c+x_{1}+x_{2}+\ldots+x_{k}
$$

where $c$ is a constant vector in $\mathbb{E}^{m}$ and $x_{1}, \ldots, x_{k}$ are non-constant $\mathbb{E}^{m}$-valued maps satisfying

$$
\Delta x_{i}=\lambda_{i} x_{i}, i=1, \ldots, k
$$

The decomposition (2.1) is called the spectral decomposition or the spectral resolution of the immersion $x$. In particular, if all of the eigenvalues $\lambda_{1}, \ldots, \lambda_{k}$ associated with the spectral decomposition are mutually different, then the immersion $x$ (or the submanifold $M$ ) is said to be of $k$-type. In particular, if one of $\lambda_{1}, \ldots, \lambda_{k}$ is zero, then the immersion is said to be of $n u l l$ $k$-type. Clearly, every submanifold of null $k$-type is non-compact. A submanifold is said to be of infinite type if it is not of finite type. In terms of finite type submanifolds, a result of [160] 
states that a submanifold of $\mathbb{E}^{m}$ is of 1-type if and only if it is either a minimal submanifold of $\mathbb{E}^{m}$ or a minimal submanifold of a hypersphere of $\mathbb{E}^{m}$.

For a spherical isometric immersion $x: M \rightarrow S_{c}^{m-1} \subset \mathbb{E}^{m}$, the immersion is called masssymmetric in $S_{c}^{m-1}$ if the center of gravity of $M$ in $\mathbb{E}^{m}$ coincides with the center $c$ of the hypersphere $S_{c}^{m-1}$ in $\mathbb{E}^{m}$.

\subsection{Minimal polynomials}

For a finite type submanifold $M$ satisfying (2.1) and (2.2), the polynomial $P$ defined by

$$
P(t)=\prod_{i=1}^{k}\left(t-\lambda_{i}\right)
$$

satisfies $P(\Delta)(x-c)=0$. This polynomial $P$ is called the minimal polynomial of $M$. For an $n$-dimensional submanifold $M$ of a Euclidean space, the mean curvature vector $H$ satisfies Beltrami's formula:

$$
\Delta x=-n H
$$

It follows from (2.4) that the minimal polynomial $Q$ also satisfies $Q(\Delta) H=0$. Conversely, if $M$ is compact and if there exists a constant vector $c$ and a nontrivial polynomial $Q$ such that $Q(\Delta)(x-c)=0$ (or $Q(\Delta) H=0$ ), then $M$ is always of finite type. This characterization of finite type submanifolds via the minimal polynomial plays an important role in the theory of finite type submanifolds.

When $M$ is non-compact, the existence of a nontrivial polynomial $Q$ satisfying $Q(\Delta) H=0$ does not guarantee $M$ to be finite type. On the other hand, if either $M$ is one-dimensional or $Q$ is a polynomial of degree $k$ with exactly $k$ distinct roots, then the existence of the polynomial $Q$ satisfying $Q(\Delta)(x-c)=0$ for some constant vector $c$ does guarantee that $M$ is of finite type [82].

\subsection{A basic formula for $\Delta H$}

The following basic formula of $\Delta H$ derived in [24, 26, 29] plays important role in the study of submanifolds of low type as well as in the study of biharmonic submanifolds:

$$
\Delta H=\Delta^{D} H+\sum_{i=1}^{n} h\left(e_{i}, A_{H} e_{i}\right)+2 \operatorname{trace}\left(A_{D H}\right)+\frac{n}{2} \operatorname{grad}\langle H, H\rangle,
$$

where $\Delta^{D}$ is the Laplace operator associated with the normal connection $D, h$ is the second fundamental form, and $\left\{e_{1}, \ldots, e_{n}\right\}$ is a local orthonormal frame of $M$. In particular, if $M$ is a hypersurface of a Euclidean space $\mathbb{E}^{n+1}$, then formula (2.5) reduces to

$$
\Delta H=\left(\Delta \alpha+\alpha\|h\|^{2}\right) \xi+2 \operatorname{trace}\left(A_{D H}\right)+\frac{n}{2} \operatorname{grad}\langle H, H\rangle,
$$


where $\alpha$ is the mean curvature and $\xi$ a unit normal vector of $M$ in $\mathbb{E}^{n+1}$.

Similar formulas hold as well if the ambient spaces is pseudo-Euclidean.

\section{4. $\delta$-invariants and ideal immersions}

Let $M$ be a Riemannian $n$-manifold. Denote by $K(\pi)$ the sectional curvature of a plane section $\pi \subset T_{p} M, p \in M$. For any orthonormal basis $e_{1}, \ldots, e_{n}$ of $T_{p} M$, the scalar curvature $\tau$ at $p$ is

$$
\tau(p)=\sum_{i<j} K\left(e_{i} \wedge e_{j}\right)
$$

Let $L$ be a $r$-subspace of $T_{p} M$ with $r \geq 2$ and let $\left\{e_{1}, \ldots, e_{r}\right\}$ be an orthonormal basis of $L$. The scalar curvature $\tau(L)$ of $L$ is defined by

$$
\tau(L)=\sum_{\alpha<\beta} K\left(e_{\alpha} \wedge e_{\beta}\right), \quad 1 \leq \alpha, \beta \leq r .
$$

For given integers $n \geq 3, k \geq 1$, we denote by $\mathscr{S}(n, k)$ the finite set consisting of $k$-tuples $\left(n_{1}, \ldots, n_{k}\right)$ of integers satisfying $2 \leq n_{1}, \cdots, n_{k}<n$ and $\sum_{j=1}^{k} n_{i} \leq n$.

Put $\mathscr{S}(n)=\cup_{k \geq 1} \mathscr{S}(n, k)$. For each $k$-tuple $\left(n_{1}, \ldots, n_{k}\right) \in \mathscr{S}(n)$, the author introduced in 1990s the Riemannian invariant $\delta\left(n_{1}, \ldots, n_{k}\right)$ by

$$
\delta\left(n_{1}, \ldots, n_{k}\right)(p)=\tau(p)-\inf \left\{\tau\left(L_{1}\right)+\cdots+\tau\left(L_{k}\right)\right\}, \quad p \in M,
$$

where $L_{1}, \ldots, L_{k}$ run over all $k$ mutually orthogonal subspaces of $T_{p} M$ such that $\operatorname{dim} L_{j}=$ $n_{j}, j=1, \ldots, k$ (cf. [51] for details).

For an $n$-dimensional submanifold of $\mathbb{E}^{m}$ and for a $k$-tuple $\left(n_{1}, \ldots, n_{k}\right) \in \mathscr{S}(n)$, the author proved the following general sharp inequality [48, 52]:

$$
\delta\left(n_{1}, \ldots, n_{k}\right) \leq \frac{n^{2}\left(n+k-1-\sum n_{j}\right)}{2\left(n+k-\sum n_{j}\right)}|H|^{2},
$$

where $|H|^{2}=\langle H, H\rangle$ denotes the squared mean curvature of $M$.

A submanifold $M$ of $\mathbb{E}^{m}$ is called $\delta\left(n_{1}, \ldots, n_{k}\right)$-ideal if it satisfies the equality case of (2.9) identically. Roughly speaking, ideal submanifolds are submanifolds which receive the least possible tension from its ambient space. For the most recent survey on $\delta$-invariants and ideal immersions, see [52, 53] for details.

\subsection{Proper and $\epsilon$-superbiharmonic submanifolds}

An immersed submanifold $M$ of a Riemannian manifold $\tilde{M}$ is said to be properly immersed if the immersion is a proper map, i.e., the preimage of each compact set in $\tilde{M}$ is compact in $M$. 
A hypersurface of a Euclidean space is called weakly convex if it has non-negative principle curvatures. Also, a hypersurface of an $(n+1)$-sphere is called isoparametric if it has constant principal curvatures.

The total mean curvature of a submanifold $M$ in a Riemannian manifold is defined to be $\int_{M}|H|^{2} d v$.

Let $M$ be a submanifold of a Riemannian manifold with inner product $\langle$,$\rangle . Then M$ is called $\epsilon$-superbiharmonic if it satisfies

$$
\langle\Delta H, H\rangle \geq(\epsilon-1)|\nabla H|^{2},
$$

where $\epsilon \in[0,1]$ is a constant.

For a complete Riemannian manifold $(N, h)$ and $\alpha \geq 0$, if the sectional curvature $K^{N}$ of $N$ satisfies

$$
K^{N} \geq-L\left(1+\operatorname{dist}_{N}\left(\cdot, q_{0}\right)^{2}\right)^{\alpha / 2}
$$

for some $L>0$ and $q_{0} \in N$, then we call that $K^{N}$ has a polynomial growth bound of order $\alpha$ from below.

\section{Finite type hypersurfaces of Euclidean space}

The class of finite type submanifolds in Euclidean spaces is huge. It includes all minimal submanifolds of Euclidean spaces, all minimal submanifolds of hyperspheres as well as all compact homogeneous submanifolds equivariantly immersed in some Euclidean space. In contrast, very few examples of finite type hypersurfaces in Euclidean spaces are known. So far, minimal surfaces, circular cylinders and the spheres are the only known surfaces of finite type in $\mathbb{E}^{3}$.

In [40], the author asked the following problem.

Problem 1. Classify all finite type hypersurfaces in $\mathbb{E}^{n+1}$. In particular, classify all finite type surfaces in $\mathbb{E}^{3}$.

This problem does have a complete solution when $n=1$. In fact, it was proved in $[25,26]$ that circles are the only finite type closed planar curves. Also, it is known that lines are the only non-closed planar curves [36]. In fact, lines are the only null finite type planar curves [56].

Next, we recall some classical results concerning Problem 1. The first result on the classification of finite type surfaces in $\mathbb{E}^{3}$ was obtained in [33] which states that circular cylinders are the only tubes of finite type. It was proved in [56] that a ruled surface in $\mathbb{E}^{3}$ is of finite type 
if and only if it is a plane, a circular cylinder or a helicoid. Furthermore, it was shown in [62] that spheres and circular cylinders are the only quadrics of finite type in $\mathbb{E}^{3}$. In [117], it was proved that a cone in $\mathbb{E}^{3}$ is of finite type if and only if it is a plane. Also, it was shown in [76] that every compact 2-type hypersurface of $\mathbb{E}^{n+1}$ has non-constant mean curvature.

For compact finite type surfaces in $\mathbb{E}^{3}$, the author made the following conjecture in [29, 40].

Conjecture 1. The only compact surfaces of finite type in $\mathbb{E}^{3}$ are the ordinary spheres.

Besides the classical results given above, there are several additional results obtained in $[3,13,33,88,94,122,164]$ which support this conjecture. However, after more than two decades Conjecture 1 remains open.

In addition to Conjecture 1, the author would like to make the following two additional conjectures which are closely related to Conjecture 1 .

Conjecture 1.A. The only surfaces of finite type in $\mathbb{E}^{3}$ are minimal surfaces, and open portions of spheres and circular cylinders.

Conjecture 1.B. The only compact hypersurfaces of finite type in Euclidean space are ordinary hyperspheres.

\section{Spherical hypersurfaces of finite type}

\subsection{Finite type spherical hypersurfaces}

In contrast to Euclidean hypersurfaces, there do exist many 1-type and 2-type spherical hypersurfaces. The author proved in [30] that every compact hypersurface of a hypersphere $S^{n+1} \subset \mathbb{E}^{n+2}$, not a small hypersphere, is mass-symmetric and of 2-type if and only if it has non-zero constant mean curvature and constant scalar curvature. Consequently, every isoparametric hypersurface of a hypersphere is either of 1-type or mass-symmetric and 2-type. Since there exist non-minimal isoparametric hypersurfaces in hyperspheres, there do exist 2-type hypersurfaces of hyperspheres.

The following problem was proposed by the author in [40].

Problem 2. Study and classify 2-type hypersurfaces in a hypersphere of $\mathbb{E}^{n+2}$. In particular, classify 2-type hypersurfaces of a hypersphere $S^{4}$ in $\mathbb{E}^{5}$.

It is known that a compact surface $M$ in $S^{3}$ is of 2-type if and only if it is the product of two plane circles of different radii, i.e., $M=S^{1}(a) \times S^{1}(b)$ with $a \neq b$ and $a^{2}+b^{2}=1[16,25]$. The same result also holds without compactness [119]. 
For 2-type hypersurfaces in $S^{4}$, we have the following classification theorem from [46]. A compact hypersurface of $S^{4}(1)$ is of 2-type if and only if it is congruent to one of the following two hypersurfaces:

(a) a standard imbedding $S^{1} \times S^{2} \subset S^{4}(1) \subset \mathbb{E}^{5}$ such that the radii $r_{1}$ of $S^{1}$ and $r_{2}$ of $S^{2}$ satisfying $r_{1}^{2}+r_{2}^{2}=1$ and $\left(r_{1}, r_{2}\right) \neq\left(\frac{1}{\sqrt{3}}, \frac{\sqrt{2}}{\sqrt{3}}\right)$

(b) a tube $T^{r}\left(V^{2}\right)$ with radius $r \neq \frac{\pi}{2}$ over the Veronese surface $V^{2}$ in $S^{4}(1)$.

It was proved by Hasanis and Vlachos in [120] that every 2-type hypersurface of a hypersphere $S^{n+1}$ has nonzero constant mean curvature and constant scalar curvature.

The following problems was also proposed by the author in [40].

Problem 3. Classify finite type hypersurfaces of a hypersphere in $\mathbb{E}^{n+2}$.

Very little were known on finite type spherical hypersurfaces with type number $\geq 3$. The only known general result in this respect is that every 3-type spherical hypersurface has nonconstant mean curvature [37, 75]. For finite type spherical surfaces in a 3-sphere $S^{3}$ with arbitrary type number, the author and Dillen proved in [61] that standard 2-spheres and products of two plane circles are the only compact finite type surfaces with constant Gauss curvature in $S^{3}$.

The classification of $k$-type spherical hypersurfaces with $k \geq 3$ remains a very challenge problem. From all available information, it seems to the author that there exist no surfaces of $k$-type in $S^{3}$ for any finite $k$ greater than 2 .

At an international conferences held at Berlin in 1990, the author proposed the following conjecture.

Conjecture 2. Minimal surfaces, standard 2-spheres and products of two plane circles are the only finite type surfaces in $S^{3} \subset \mathbb{E}^{4}$.

This conjecture was also proposed in [40]. There are several results obtained in [37, 55, $75,110,146]$ which support this conjecture. On the other hand, this conjecture remains open in general.

\subsection{Dupin hypersurfaces}

A hypersurface $M$ of $S^{n+1}$ is called a Dupin hypersurface if the multiplicities of the principal curvatures are constant and each principal curvature are constant along its principal directions. Since 2-type spherical hypersurfaces are mass-symmetric [120], a result of [30] 
implies that if a compact Dupin hypersurface $M$ of $S^{n+1}$ is not of 1-type and if it has at most three distinct principal curvatures, then it is parametric if and only if it is of 2-type.

For Dupin hypersurfaces, the author gave the following open problem in [40].

Problem 4. When is a Dupin hypersurface of a hypersphere to be offinite type? When is a finite type Dupin hypersurface of a hypersphere to be isoparametric?

As far as the author know, no further results were known for this problem.

\section{Spherical 2-type submanifolds of higher codimension}

It is well-known that there exist ample examples of 1-type surfaces lying fully in odddimensional spheres as well as in even-dimensional spheres. Also, it is known that there exist abundant examples of mass-symmetric 2-type surfaces lying fully in odd-dimensional hyperspheres (see, e.g., [14, 26, 116, 136, 143]). In contrast, it was proved by the author and Barros in [14] that there do not exist mass-symmetric 2-type surfaces lying fully in $S^{4}$. So far there are no known examples of non-mass-symmetric 2-type surfaces in $S^{4}$. In this respect, the author asked in [40] the following

Problem 5. Do there exist non-mass-symmetric 2 -type surfaces in $S^{4}$ ?

Since there exist no known examples of mass-symmetric 2-type surfaces lying fully in a hypersphere of a Euclidean space for any even codimension, the author proposed the following problem in [40] which is more general than Problem 5.

Problem 6. Do there exist 2-type surfaces lying fully in an even-dimensional hypersphere of a Euclidean space? In particular, do there exist mass-symmetric 2-type surfaces lying fully in an even-dimensional hypersphere?

If the mass-symmetric, 2-type, spherical surfaces come from one of the following four families

- stationary surfaces [14],

- topological 2-spheres[136],

- surfaces with constant Gauss curvature [143],

- flat Chen surfaces [116],

the answers to Problem 6 is known to be negative.

Up to author's knowledge, Problems 5 and 6 remain unsolved. 


\section{Linearly independent submanifolds}

The notion of linearly independent immersions or submanifolds were introduced by the author in [39]. Suppose that $x: M \rightarrow \mathbb{E}^{m}$ is a $k$-type immersion whose spectral decomposition is given by (2.1). Denote by $E_{i}$ the subspace of $\mathbb{E}^{m}$ spanned by $\left\{x_{i}(p), p \in M\right\}, i \in\{1, \ldots, k\}$. The immersion $x$ (or the submanifold $M$ ) is called linearly independent if the subspaces $E_{1}, \ldots, E_{k}$ are linearly independent. The immersion $x$ is called orthogonal if the subspaces $E_{1}, \ldots, E_{k}$ are mutually orthogonal.

Clearly, every orthogonal immersion is linearly independent and every 1-type immersion is orthogonal. There exist many examples of orthogonal immersions and abundant examples of linearly independent immersions which are not orthogonal. In fact, every $k$-type curve lying fully in $\mathbb{E}^{2 k}$ and every null $k$-type curve lying fully in $\mathbb{E}^{2 k-1}$ are linearly independent curves; but $W$-curves are the only orthogonal curves in a Euclidean space.

For a given linearly independent immersion $x: M \rightarrow \mathbb{E}^{m}$ and a given point $p \in M$, one has the notion of the adjoint hyperquadric $Q_{p}$ defined in [39]. When $M$ lies in one of the adjoint hyperquadrics $Q_{p}, p \in M$, then all of the adjoint hyperquadrics $Q_{p}, p \in M$, coincide. This common hyperquadric is called the adjoint hyperquadric of the linearly independent immersion [39].

It was shown in [39] that if $x: M \rightarrow \mathbb{E}^{m}$ is a linearly independent immersion of a compact manifold, then the submanifold lies in its adjoint hyperquadric if and only if the submanifold is spherical with an appropriate center. Moreover, it is known that a non-minimal linearly independent immersion $x$ is orthogonal if and only if $M$ is immersed by $x$ as a minimal submanifold of the adjoint hyperquadric [39]. Consequently, every orthogonal immersion of a compact manifold is spherical. Moreover, it also known that each compact homogeneous submanifold, equivariantly immersed in $\mathbb{E}^{m}$, is orthogonal and therefore it is immersed as a minimal submanifold in its adjoint hyperquadric [39].

It is known that the only linearly independent Euclidean hypersurfaces are hyperspheres, minimal hypersurfaces or spherical hypercylinders (see [66, 82, 94, 118, 122]). Also, by applying the classification theorem of 2-type curves in Euclidean space from [64], one may conclude that the only linearly independent curves of codimension 2 in a Euclidean space are circles, lines and circular helices. In this respect, the author would like to point out that there do exist abundant examples of linearly independent curves of codimension 3 in Euclidean space.

In [40] the next two problems on linearly independent immersions were proposed.

Problem 7. Study and classify linearly independent2-type immersions.

Problem 8. Study and classify linearly independent submanifolds of codimension 2. 
In [128], Jang studied linearly independent immersions with codimension $\leq 3$. He derived some necessary and sufficient conditions for linearly independent immersions with codimension $\leq 3$ to be orthogonal. His results provide some partial generalizations of author's results in [39] for codimension $\leq 3$.

The class of linearly independent immersions lies in a much larger class of immersions; namely, the class of immersions of restricted type introduced in [67].

A submanifold of a Euclidean space is said to be of restricted type if its shape operator with respect to the mean curvature vector is the restriction of a fixed linear transformation of the ambient space to the tangent space of the submanifold at every point of the submanifold. There are very few known results on submanifolds of restricted type. The only known classification results for submanifolds of restricted in Euclidean spaces are the classifications of planar curves and Euclidean hypersurface of restricted type [67] (see also [163, 164]).

\section{Null 2-type submanifolds}

It was proved in [75] that every 2-type submanifold in a Euclidean space with parallel mean curvature vector is either spherical or of null 2-type. Related with this the author also asked in [40] the following.

Problem 9. Is every $n$-dimensional non-null2-type submanifold with constant mean curvature be in $\mathbb{E}^{n+2}$ spherical?

It follows from the definition of null 2-type submanifolds and formula (2.3) that the mean curvature vector $H$ of a null 2-type submanifold satisfies

$$
\Delta H=\lambda H,
$$

where $\lambda$ is a nonzero real number. It was proved in [36] that biharmonic submanifolds, null 2-type submanifolds and 1-type submanifolds are the only Euclidean submanifolds satisfying (7.1).

From the classification of finite type planar curves, we know that there do not exist null 2-type planar curves. Furthermore, it follows from [36] that the only null 2-type curves in Euclidean spaces are circular helices with nonzero torsion in $\mathbb{E}^{3}$. A general result from [35] states that circular cylinders are the only null 2 type surfaces in Euclidean 3-space. Since biharmonic and null 2-type surfaces in $\mathbb{E}^{3}$ were classified, the complete classification of surfaces in $\mathbb{E}^{3}$ satisfying (7.1) was done.

Next, we present some classical results on null 2-type Euclidean surfaces with codimension $\geq 2$. It is known in [36] that null 2-type surfaces in $\mathbb{E}^{4}$ are helical cylinders if they have 
constant mean curvature. However, it still unknown whether every null 2-type surface in $\mathbb{E}^{4}$ has constant mean curvature.

As a generalization of [35], null 2-type hypersurfaces with at most two distinct principal curvatures were classified in [110] (see [109] for null 2-type conformally flat hypersurfaces of dimension $\neq 3$ ). In [36], the author proved that a surface $M$ in $\mathbb{E}^{4}$ is of null 2-type with parallel normalized mean curvature vector if and only if it is an open portion of a circular cylinder in a hyperplane of $\mathbb{E}^{4}$. The author also proved that the only null 2-type surfaces in $\mathbb{E}^{4}$ with constant mean curvature are open portion of helical cylinders. Hasani and Vlachos proved in [124] that non-spherical hypersurfaces in $\mathbb{E}^{4}$ with non-vanishing constant mean curvature and constant scalar curvature are the only null 2-type hypersurfaces.

For null 2-type submanifolds, the author proposed in [40] the following.

Problem 10. Study and classify null2-type submanifolds. In particular, classify all null2-type surfaces in 4-dimensional Euclidean space and in 4-dimensional pseudo-Euclidean spaces.

Now, we present some later development concerning Problem 10. It was showed by Li in [130] that a surface in $\mathbb{E}^{m}$ with parallel normalized mean curvature vector is of null 2-type if and only if it is an open portion of a circular cylinder. Also, it was proved in [131] that, for a non-pseudo-umbilical Chen surface $M$ in $\mathbb{E}^{m}$, if $M$ is of null 2-type and with constant mean curvature, then $M$ is flat and must lie in a totally geodesic $\mathbb{E}^{6} \subset \mathbb{E}^{m}$.

In [103], Dursun classified 3-dimensional null 2-type submanifold of $\mathbb{E}^{5}$ with two distinct principal curvatures in the parallel mean curvature direction and with constant squared norm of the second fundamental form. Also, he proved in [105] that if a null 2-type submanifold of $\mathbb{E}^{n+2}$ with codimension 2 has flat normal connection, constant mean curvature and nonparallel mean curvature vector, then the first normal space must one-dimensional. By using this fact, he derived some some classification results.

Recently, it was proved by the author and Garay in [70] that a null 2-type hypersurface of $\mathbb{E}^{n+1}$ is $\delta(2)$-ideal if and only if it an open portion of a spherical cylinder $S^{n-1} \times \mathbb{E}$.

However, until now there are still no complete classification of null 2-type submanifolds. In particular, null 2-type surfaces in Euclidean 4-space $\mathbb{E}^{4}$ are not completely classified.

\section{Finite type submanifolds in homogeneous spaces}

For finite type submanifolds of compact irreducible homogeneous manifolds, the following problem was proposed in [40].

Problem 11. Let $\tilde{M}$ be a compact irreducible homogeneous manifold immersed in a Euclidean space $\mathbb{E}^{N}$ by its first standard immersion $\phi$ and $M$ a submanifold of $\tilde{M}$. When $M$ is of finite type 
in $\mathbb{E}^{N}$ via $\phi$ ? In particular, when $M$ is of 1 - or 2 -type in $\mathbb{E}^{N}$ via $\phi$ ?

If $\tilde{M}$ is a projective $m$-space $F P^{m}$ over a field $F=\mathbb{R}, \mathbb{C}$ or $\mathbb{U}$ with a standard Riemannian metric, this problem has been investigated in [15, 18, 26, 27, 142, 161], [155]-[157] and [97][102], among others. When $\tilde{M}$ is the real Grassmannian $G^{R}(p, q)$ or the space $U(n) / O(n)$, this problem has been investigated in [19].

Several recent results in this respect were given by Dimitrić in a series of his papers [99][102]. In [102] he studied 2 and 3-type Hopf hypersurfaces of complex projective space $C P^{m}$ and of complex hyperbolic space $\mathrm{CH}^{m}$ via some suitable imbeddings into pseudo-Euclidean spaces of Hermitian matrices. He proved in [102] that tubes of certain radii around totally geodesic $C P^{k} \subset C P^{m}, k \in\{0, \ldots, m-1\}$, and around the complex quadric $Q^{m-1} \subset C P^{m}$ are 2-type Hopf hypersurfaces in $C P^{m}$. Conversely, every 2-type Hopf hypersurface in $C P^{m}$ is locally congruent to such a tube. For $C H^{m}$, he shown that a Hopf hypersurface is of 2-type if and only if it is locally congruent to a geodesic hypersphere or to a tube of any radius $r>0$ around a totally geodesic $\mathrm{CH}^{m-1} \subset \mathrm{CH}^{m}$. He also obtained partial classification of 3-type Hopf hypersurfaces in $C P^{2}$ as well as in $C H^{2}$.

\section{Biharmonic submanifolds}

The study of biharmonic submanifolds was initiated in the middle of 1980s via author's study of finite type submanifolds; also independently by Jiang [129] via his study of EulerLagrange's equation of bienergy functional in the sense of Eells and Lemaire [106, 107].

Let $x: M \rightarrow \mathbb{E}^{m}$ be an isometric immersion. As we already mentioned earlier, the position vector field of $M$ in $\mathbb{E}^{m}$ satisfies Beltrami's formula:

$$
\Delta x=-n H .
$$

Formula (9.1) implies that the immersion is minimal if and only if it is harmonic, i.e., $\Delta x=0$. An immersion $x: M \rightarrow \mathbb{E}^{m}$ is called biharmonic if

$$
\Delta^{2} x=0, \quad \text { orequivalently } \Delta H=0,
$$

holds identically.

Let $x: M \rightarrow \mathbb{E}^{m}$ be an isometric immersion. It follows from (2.5) and (9.2) that $M$ is a biharmonic submanifold if and only if it satisfies the following fourth order strongly elliptic semi-linear PDE system (see, e.g., [24, 52]):

$$
\begin{aligned}
& \Delta^{D} H+\sum_{i=1}^{n} \sigma\left(A_{H} e_{i}, e_{i}\right)=0, \\
& n \operatorname{grad}\langle H, H\rangle+4 \operatorname{trace} A_{D H}=0,
\end{aligned}
$$


where $\left\{e_{1}, \ldots, e_{n}\right\}$ is an orthonormal frame of $M$.

It is obvious that minimal immersions are trivially biharmonic. Thus, the real problem is if there are other submanifolds besides minimal ones that are biharmonic.

In [40] the author made the following simple geometric question.

Problem 12. Other than minimal submanifolds, which submanifolds of $\mathbb{E}^{m}$ are biharmonic?

A biharmonic map is a map $\phi:(M, g) \rightarrow(N, h)$ between Riemannian manifolds that is a critical point of the bienergy functional:

$$
E^{2}(\phi, D)=\frac{1}{2} \int_{D}\left\|\tau_{\phi}\right\|^{2} * 1
$$

for every compact subset $D$ of $M$, where $\tau_{\phi}=\operatorname{trace}_{g} \nabla d \phi$ is the tension field $\phi$.

The Euler-Lagrange equation of (9.5) gives the following biharmonic map equation [129]:

$$
\tau_{\phi}^{2}:=\operatorname{trace}_{g}\left(\nabla^{\phi} \nabla^{\phi}-\nabla_{\nabla^{M}}^{\phi}\right) \tau_{\phi}-\operatorname{trace}_{g} R^{N}\left(d \phi, \tau_{\phi}\right) d \phi=0,
$$

where $R^{N}$ denotes the curvature tensor of $(N, h)$. Equation (9.6) implies that $\phi$ is a biharmonic map if and only if its bi-tension field $\tau_{\phi}^{2}$ vanishes.

For an $n$-dimensional submanifold $M$ of $\mathbb{E}^{m}$, if we denote by $\iota: M \rightarrow \mathbb{E}^{m}$ the inclusion map of the submanifold, then the tension field of the inclusion map is given by $\tau_{\iota}=-\Delta \iota=-n H$ according to Beltrami's formula. Therefore, the submanifold $M$ is biharmonic if and only if

$$
n \Delta H=-\Delta^{2} \iota=-\tau_{\iota}^{2}=0
$$

i.e., the inclusion map $\iota$ is a biharmonic map.

\section{Biharmonic conjectures}

\subsection{The original biharmonic conjecture}

The author shown in 1985 that biharmonic surfaces in $\mathbb{E}^{3}$ are minimal (independently by Jiang [129]). This result was the starting point of Dimitrić's work on his thesis [95] at Michigan State University. In [95], Dimitrić extended author's result (unpublished then) to biharmonic Euclidean hypersurfaces with at most two distinct principal curvatures [95]. He also proved that each biharmonic submanifold of finite type in in a Euclidean space is minimal. Furthermore, he proved that biharmonic Euclidean submanifolds of finite type and pseudo-umbilical biharmonic Euclidean submanifolds are always minimal.

In [40], the author pointed out that spherical biharmonic submanifolds of a Euclidean space are minimal. Moreover, it was proved by Hasani and Vlachos in [123] that biharmonic hypersurfaces of $\mathbb{E}^{4}$ are also minimal. 
In [40], the author proposed the following Biharmonic Conjecture.

Conjecture 3: The only biharmonic submanifolds of Euclidean spaces are the minimal ones.

\subsection{Generalized Chen's biharmonic conjectures}

Caddeo, Montaldo and Oniciuc proved in [21] that every biharmonic surface in the hyperbolic 3-space $H^{3}$ is minimal. They also proved in [20] that biharmonic hypersurfaces of $H^{n}$ with at most two distinct principal curvatures are minimal. Based on these facts, they made the following conjecture in [20].

Generalized Chen's Conjecture: Every biharmonic submanifold of a Riemannian manifold with non-positive sectional curvature is minimal.

The study of biharmonic submanifolds is nowadays a very active research subject. Biharmonic submanifolds have received a growing attention with many progresses done since the beginning of this century.

\section{Recent developments on author's original biharmonic conjecture}

From the definition of biharmonic submanifolds and Hopf's lemma we see that biharmonic submanifolds in a Euclidean space are always non-compact.

The following recent results provide strong supports to the original biharmonic conjecture.

- Biharmonic properly immersed [138].

- Biharmonic submanifolds which are complete and proper [1].

- $\delta(2)$-ideal and $\delta$ (3)-ideal biharmonic hypersurfaces [79].

- Weakly convex biharmonic submanifolds [133].

- Submanifolds whose $L^{p}, p \geq 2$, integral of the mean curvature vector field satisfies certain decay condition at infinity [135].

- Biharmonic submanifolds satisfying the decay condition at infinity

$$
\lim _{\rho \rightarrow \infty} \frac{1}{\rho^{2}} \int_{f^{-1}\left(B_{\rho}\right)}|H|^{2} d v=0,
$$

where $f$ is the immersion, $B_{\rho}$ is a geodesic ball of $N$ with radius $\rho$ [167].

The author would like to point out that Y.-L. Ou showed recently in [150] that the original biharmonic conjecture cannot be generalized to biharmonic conformal submanifolds in Euclidean spaces. 
Remark 1. Conjecture 3 remains open.

Remark 2. Conjecture 3 is false if the ambient Euclidean space were replaced by a pseudoEuclidean space. The simplest examples are constructed in [71].

\section{Recent developments on generalized biharmonic conjecture}

In the last few years, there are many partial answers support the generalized Chen's biharmonic conjecture. The following is a list of recent results which support the generalized biharmonic conjecture.

- Biharmonic hypersurfaces in $H^{4}(-1)$ [11].

- Pseudo-umbilical biharmonic submanifolds of $H^{m}(-1)$ [20].

- Totally umbilical biharmonic hypersurfaces in Einstein spaces [151].

- Biharmonic hypersurfaces with finite total mean curvature in a Riemannian manifold of non-positive Ricci curvature [147].

- Biharmonic submanifolds with finite total mean curvature in a Riemannian manifold of non-positive sectional curvature [148].

- Biharmonic properly immersed submanifolds in a complete Riemannian manifold with non-positive sectional curvature whose sectional curvature has polynomial growth bound of order less than 2 from below [139].

- Complete biharmonic submanifolds with finite bi-energy and energy in a non-positively curved Riemannian manifold [149].

- Complete oriented biharmonic hypersurfaces $M$ whose mean curvature $H$ satisfying $H \in$ $L^{2}(M)$ in a Riemannian manifold with non-positive Ricci tensor [2].

- Compact biharmonic submanifolds in a Riemannian manifold with non-positive sectional curvature [140].

- Complete biharmonic submanifolds (resp., hypersurfaces) in a Riemannian manifold whose sectional curvature (resp., Ricci curvature) is non-positive with at most polynomial volume growth [134].

- Complete biharmonic submanifolds (resp., hypersurfaces) in a negatively curved Riemannian manifold whose sectional curvature (resp., Ricci curvature) is smaller that $-\epsilon$ for some $\epsilon>0$ [134].

- Complete biharmonic submanifolds (resp., hypersurfaces) $M$ in a Riemannian manifold of non-positive sectional (resp., Ricci) curvature whose mean curvature vector satisfies $\int_{M}\left|H^{p}\right| d v<\infty$ for some $p>0[134]$. 
- Complete biharmonic hypersurfaces $M$ in a Riemannian manifold of non-positive Ricci curvature whose mean curvature vector satisfies $\int_{M}|H|^{\alpha} d \nu<\infty$ for some $\epsilon>0$ with $1+\epsilon \leq$ $\alpha<\infty$ [140].

- $\epsilon$-superbiharmonic submanifolds in a complete Riemannian manifolds satisfying the decay condition at infinity

$$
\lim _{\rho \rightarrow \infty} \frac{1}{\rho^{2}} \int_{f^{-1}\left(B_{\rho}\right)}|H|^{2} d v=0,
$$

where $f$ is the immersion, $B_{\rho}$ is a geodesic ball of $N$ with radius $\rho$ [167].

- Proper $\epsilon$-superharmonic submanifolds $M$ with $\epsilon>0$ in a complete Riemannian manifold $N$ whose mean curvature vector satisfying the growth condition

$$
\lim _{\rho \rightarrow \infty} \frac{1}{\rho^{2}} \int_{f^{-1}\left(B_{\rho}\right)}|H|^{2+a} d v=0,
$$

where $f$ is the immersion, $B_{\rho}$ is a geodesic ball of $N$ with radius $\rho$, and $a \geq 0$ [134].

On the other hand, Ou and Tang [153] proved that generalized biharmonic conjecture is false in general by constructing foliations of proper biharmonic hyperplanes in some conformally flat 5-manifolds with negative sectional curvature. Further counterexamples to the generalized biharmonic conjecture were constructed in [132].

Finally, the author would like to recall the following two biharmonic conjectures mentioned earlier in [54] which are closely related to author's original biharmonic conjecture.

Biharmonic Conjecture for Hypersurfaces: Every biharmonic hypersurface of Euclidean spaces is minimal.

The global version of author's original biharmonic conjecture can be found, for instance, in $[1,140]$.

Global Version of Chen's biharmonic Conjecture: Every complete biharmonic submanifold of a Euclidean space is minimal.

\section{References}

[1] K. Akutagawa and S. Maeta, Biharmonic properly immersed submanifolds in Euclidean spaces, Geom. Dedicata, 164 (2013), 351-355.

[2] L. J. Alías, S. C. García-Martínez and M. Rigoli, Biharmonic hypersurfaces in complex Riemannian manifolds, Pacific J. Math., 263 (2013), 1-12.

[3] J. Arroyo, O. J. Garay and J. J. Mencía, On a family of surfaces of revolution of finite Chen-type, Kodai Math. J., 21 (1998), 73-80. 
[4] K. Arslan, R. Ezentas, C. Murathan and T. Sasahara, Biharmonic anti-invariant submanifolds in Sasakian space forms, Beiträge Algebra Geom., 48 (2007), 191-207.

[5] A. Arvanitoyeorgos, G. Kaimakamis and D. Palamourdas, Chen's conjecture and generalizations, Symp. Diff. Geom. Submanifolds, 109-112, 2007.

[6] C. Baikoussis, D. E. Blair, B. Y. Chen and F. Defever, Hypersurfaces of restricted type in Minkowski space, Geom. Dedicata, 62 (1996), 318-332.

[7] C. Baikoussis, B. Y. Chen and L. Verstraelen, Ruled surfaces and tubes with finite type Gauss maps, Tokyo J. Math., 16 (1993), 341-349.

[8] A. Balmuş, S. Montaldo and C. Oniciuc, Classification results for biharmonic submanifolds in spheres, Israel J. Math., 168 (2008), 201-220.

[9] A. Balmuş, S. Montaldo and C. Oniciuc, Classification results and new examples of proper biharmonic submanifolds in spheres, Note Mat., 1 (2008), suppl. no. 1, 49-61.

[10] A. Balmuş, S. Montaldo and C. Oniciuc, Properties of biharmonic submanifolds in spheres, J. Geom. Symmetry Phys., 17 (2010), 87-102.

[11] A. Balmuş, S. Montaldo and C. Oniciuc, Biharmonic hypersurfaces in 4-dimensional space forms, Math. Nachr., 283 (2010), 1696-1705.

[12] A. Balmuş, S. Montaldo and C. Oniciuc, New results toward the classification of biharmonic submanifolds in $S^{n}$, An. St. Univ. Ovidius Constanta, 20 (2012), 89-114.

[13] M. Barros, There exist no 2-type surfaces in $E^{3}$ which are images under stereographic projection of minimal surfaces in $S^{3}$, Ann. Global Anal. Geom., 10 (1992), 219-226.

[14] M. Barros and B. Y. Chen, Stationary 2-type surfaces in a hypersphere, J. Math. Soc. Japan, 39 (1987), 627-648.

[15] M. Barros and B. Y. Chen, Spherical submanifolds which are of 2-type via the second standard immersion of the sphere, Nagoya Math. J., 108 (1987), 77-91.

[16] M. Barros and O. J. Garay, 2-type surfaces in $S^{3}$, Geometriae Dedicata, 24 (1987), 329-336.

[17] M. Barros and O. J. Garay, On submanifolds with harmonic mean curvature, Proc. Amer. Math. Soc., 123 (1996), 545-2549.

[18] M. Barros and F. Urbano, Spectral geometry of minimal surfaces in the sphere, Tohoku Math. J., 39 (1987), 575-588.

[19] D. Brada and L. Niglio, Connected compact minimal Chen type-1 submanifolds of Grassmannian manifold, Bull. Soc. Math. Belg. Sér. B., 44 (1992), 299-310.

[20] R. Caddeo, S. Montaldo and and C. Oniciuc, Biharmonic submanifolds of $S^{3}$, Internat. J. Math., 12 (2001), 867-876.

[21] R. Caddeo, S. Montaldo and and C. Oniciuc, Biharmonic submanifolds in spheres, Israel J. Math., 130 (2002), 109-123.

[22] R. Caddeo, S. Montaldo and P. Piu, On biharmonic maps, Contemp. Math., 288 (2001), $286-290$.

[23] B. Y. Chen, On the total curvature of immersed manifolds, IV: Spectrum and total mean curvature, Bull. Inst. Math. Acad. Sinica, 7 (1979), 301-311.

[24] B. Y. Chen, On the total curvature of immersed manifolds, VI: Submanifolds of finite type and their applications, Bull. Inst. Math. Acad. Sinica, 11 (1983), 309-328.

[25] B. Y. Chen, On submanifolds of finite type, Soochow J. Math., 9 (1983), 65-81.

[26] B. Y. Chen, Total Mean Curvature and Submanifolds of Finite Type, World Scientific Publisher, 1984.

[27] B. Y. Chen, On the first eigenvalue of Laplacian of compact minimal submanifolds of rank one symmetric spaces, Chinese J. Math., 11 (1983), 259-273.

[28] B. Y. Chen, Finite type submanifolds in pseudo-Euclidean spaces and applications, Kodai Math. J., 8 (1985), 358-374.

[29] B. Y. Chen, Finite Type Submanifolds and Generalizations, University of Rome, Rome, 1985.

[30] B. Y. Chen, 2-type submanifolds and their applications, Chinese J. Math., 14 (1986), 1-14.

[31] B. Y. Chen, Finite type pseudo-Riemannian submanifolds, Tamkang J. Math., 17 (1986), 137-151.

[32] B. Y. Chen, Some estimates of total tension and their applications, Kodai Math. J., 10 (1987), 93-101. 
[33] B. Y. Chen, Surfaces of finite type in Euclidean 3-space, Bull. Soc. Math. Belg. Ser. B, 39 (1987), $243-254$.

[34] B. Y. Chen, Mean curvature of 2-type spherical submanifolds, Chinese J. Math., 16 (1988), 1-9.

[35] B. Y. Chen, Null 2-type surfaces in $E^{3}$ are circular cylinders, Kodai Math. J., 11 (1988), 295-299.

[36] B. Y. Chen, Null 2-type surfaces in Euclidean space, Algebra, Analysis and Geometry, (1988), 1-18.

[37] B. Y. Chen, 3-type surfaces in $S^{3}$, Bull. Soc. Math. Belg. Sér. B, 42 (1990), 379-381.

[38] Chen, B. Y., Local rigidity theorems of 2-type hypersurfaces in a hypersphere, Nagoya Math. J., 122 (1991), 139-148.

[39] Chen, B. Y., Linearly independent, orthogonal, and equivariant immersions, Kodai Math. J., 14 (1991), 341349.

[40] B. Y. Chen, Some open problems and conjectures on submanifolds of finite type, Soochow J. Math., 17 (1991), 169-188.

[41] B. Y. Chen, Submanifolds of finite type in hyperbolic spaces, Chinese J. Math., 20 (1992), 5-21.

[42] B. Y. Chen, Some pinching and classification theorems for minimal submanifolds, Arch. Math., 60 (1993), 568-578.

[43] B. Y. Chen, Classification of tensor product immersions which are of 1-type, Glasgow Math. J., 36 (1994), 255264.

[44] B. Y. Chen, Some classification theorems for submanifolds in Minkowski space-time, Archiv der Math., 62 (1994), 177-182.

[45] B. Y. Chen, Tubular hypersurfaces satisfying a basic equality, Soochow J. Math., 20 (1994), 569-586.

[46] B. Y. Chen, A Riemannian invariant for submanifolds in space forms and its applications, Geometry and topology of submanifolds, VI, 58-81, 1994.

[47] B. Y. Chen, Submanifolds in de Sitter space-time satisfying $\Delta H=\lambda H$, Iseral J. Math., 89 (1995), $373-391$.

[48] B. Y. Chen, A report on submanifolds of finite type, Soochow J. Math., 22 (1996), 117-337.

[49] B. Y. Chen, Some new obstruction to minimal and Lagrangian isometric immersions, Japan. J. Math., 26 (2000), 105-127.

[50] B. Y. Chen, Riemannian submanifolds, Handbook of Differential Geometry, vol. 1, North Holland, Amsterdam, 187-418, 2000.

[51] B. Y. Chen, Submanifolds with parallel mean curvature vector in Riemannian and indefinite space forms, Arab J. Math. Sci., 16 (2010), 1-46.

[52] B. Y. Chen, Pseudo-Riemannian Geometry, $\delta$-invariants and Applications, World Scientific, Hackensack, NJ, 2011.

[53] B. Y. Chen, A tour through $\delta$-invariants: From Nash embedding theorem to ideal immersions, best ways of living and beyond, Publ. Inst. Math. (Beograd) (N.S.), 94(108) (2013), 67-80.

[54] B. Y. Chen, Recent developments of biharmonic conjectures and modified biharmonic conjectures, Pure and Applied Differential Geometry - PADGE 2012, 81-90, Shaker Verlag, Aachen, 2013.

[55] B. Y. Chen, M. Barros and O. J. Garay, Spherical finite type hypersurfaces, Algebras Groups Geom., 4 (1987), $58-72$.

[56] B. Y. Chen, J. Deprez, F. Dillen, L. Verstraelen and L. Vrancken, Finite type curves, Geometry and Topology of Submanifolds, II, 76-110, 1990.

[57] B. Y. Chen, J. Deprez and Verheyen, P., Immersions, dans un espace euclidien, d'un espace symétrique compact de rang un à géodésiques simples, C. R. Acad. Sc. Paris, 304 (1987), 567-570.

[58] B. Y. Chen, J. Deprez and P. Verheyen, Immersions with many circular geodesics, Geometry and Topology of Submanifolds IV, 111-132, 1992.

[59] B. Y. Chen, J. Deprez and P. Verheyen, Immersions with geodesics of 2-type, Geometry and Topology of Submanifolds IV, 87-110, 1992.

[60] B. Y. Chen, J. Deprez and P. Verheyen, A note on the centroid set of compact symmetric spaces, Geometry and Topology of Submanifolds IV, 3-10, 1992.

[61] B. Y. Chen and F. Dillen, Surfaces of finite type and constant curvature in the 3-sphere, C. R. Math. Rep. Acad. Sci. Canada, 12 (1990), 47-49. 
[62] B. Y. Chen and F. Dillen, Quadrics of finite type, J. Geometry, 38 (1990), 16-22.

[63] B. Y. Chen, F. Dillen and H. Song, Quadric hypersurfaces of finite type, Colloq. Math., 63 (1992), $145-152$.

[64] B. Y. Chen, F. Dillen and L. Verstraelen, Finite type space curves, Soochow J. Math., 12 (1986), 1-10.

[65] B. Y. Chen, F. Dillen, L. Verstraelen and L. Vrancken, Ruled surfaces of finite type, Bull. Austral. Math. Soc., 42 (1990), 447-453.

[66] B. Y. Chen, F. Dillen, L. Verstraelen and L. Vrancken, A variational minimal principle characterizes submanifolds of finite type, C.R. Acad. Sc. Paris, 317 (1993), 961-965.

[67] B. Y. Chen, F. Dillen, L. Verstraelen and L. Vrancken, Submanifolds of restricted type, J. of Geometry, 46 (1993), 20-32 .

[68] B. Y. Chen, F. Dillen, L. Verstraelen and L. Vrancken, A variational minimal principle and its applications, Kyungpook Math. J., 35 (1995), no. 3, Speical Issue, 435-444.

[69] B. Y. Chen, F. Dillen, L. Verstraelen and L. Vrancken, Compact hypersurfaces determined by a spectral variational principle, Kyushu J. Math., 49 (1995), 103-121.

[70] B. Y. Chen and O. J. Garay, $\delta(2)$-ideal null 2-type hypersurfaces of Euclidean space are spherical cylinders, Kodai Math. J., 35 (2012), 382-391.

[71] B. Y. Chen and S. Ishikawa, Biharmonic surfaces in pseudo-Euclidean spaces, Mem. Fac. Sci. Kyushu Univ. A, 45 (1991), 323-347.

[72] B. Y. Chen and S. Ishikawa, Biharmonic pseudo-Riemannian submanifolds in pseudo-Euclidean spaces, Kyushu J. Math., 52 (1998), 167-185.

[73] B. Y. Chen and S. Ishikawa, On classification of some surfaces of revolution of finite type, Tsukuba J. Math., 17 (1993), 287-298.

[74] B. Y. Chen and W. E. Kuan, The cubic representation of a submanifold, Beiträge zur Algebra und Geometrie, 35 (1994), 55-66.

[75] B. Y. Chen and S. J. Li, 3-type hypersurfaces in a hypersphere, Bull. Soc. Math. Belg. Sér. B, 43 (1991), $135-141$.

[76] B. Y. Chen and H. S. Lue, Some 2-type submanifolds and applications, Ann. Fac. Sc. Toulouse Math. Ser. V, 9 (1988), 121-131.

[77] B. Y. Chen, J.-M. Morvan and T. Nore, Énergie, tension et order des applications à valeurs dans un espace euclidien, C. R. Acad. Sc. Paris, 301 (1985), 123-126.

[78] B. Y. Chen, J.-M. Morvan and T. Nore, Energy, tension and finite type maps, Kodai Math. J., 9 (1986), $406-418$.

[79] B. Y. Chen and M. I. Munteanu, Biharmonic ideal hypersurfaces in Euclidean spaces, Differential Geom. Appl., 31 (2013), 1-16.

[80] B. Y. Chen and T. Nagano, Harmonic metrics, harmonic tensors, and Gauss maps, J. Math. Soc. Japan, 36 (1984), 295-313.

[81] B. Y. Chen and K. Ogiue, On totally real submanifolds, Trans. Amer. Math. Soc., 193 (1974), 257-266.

[82] B. Y. Chen and M. Petrovic, On spectral decomposition of immersions of finite type, Bull. Austral. Math. Soc., 44 (1991), 117-129.

[83] B. Y. Chen and P. Piccinni, Submanifolds with finite type Gauss map, Bull. Austral. Math. Soc., 44 (1987), 161-186.

[84] B. Y. Chen and H. Song, Null 2-type surfaces in Minkowski space-time, Algebras Groups Geom., 6 (1989), 333-352.

[85] B. Y. Chen and H. Song, Null 2-type surfaces in Minkowski space-time, II, Atti Acad. Peloritana Pericolanti Cl. Sci. Fis. Mat. Natur., 68 (1989), 1-12.

[86] B. Y. Chen and L. Verstraelen, Laplace Transformations of Submanifolds, Centre for Pure and Applied Differential Geometry (PADGE), vol. 1. Brussels-Leuven, 1995.

[87] F. Defever, Hypersurfaces of $\mathbb{E}^{4}$ with harmonic mean curvature vector, Math. Nachr., 196 (1998), 61-69.

[88] F. Defever, R. Deszez and L. Verstraelen, L., The compact cylcides of Dupin and a conjecture of B.-Y. Chen, J. Geometry, 46 (1993), 33-38.

[89] F. Defever, R. Deszez and L. Verstraelen, The Chen-type of noncompact cylcides of Dupin, Glasgow Math. J., 36 (1994), 71-75. 
[90] J. Deprez, Immersions of finite type of compact homogeneous Riemannian manifolds, Doctoral Thesis, Katholieke Universiteit Leuven, 1988.

[91] J. Deprez, Immersions of finite type, Geometry and Topology of Submanifolds II, 111-133, 1990.

[92] J. Deprez, F. Dillen and L. Vrancken, Finite type curves on quadrics, Chinese J. Math., 18 (1990), 95-121.

[93] F. Dillen, Ruled submanifolds of finite type, Proc. Amer. Math. Soc., 114 (1992), 795-798.

[94] F. Dillen, J. Pas, and L. Verstraelen, On surfaces of finite type in Euclidean 3-space, Kodai Math. J. 13 (1990), $10-21$.

[95] I. Dimitrić, Quadric representation and submanifolds of finite type, Ph.D. Thesis, Michigan State University, 1989.

[96] I. Dimitrić, Submanifolds of $E^{m}$ with harmonic mean curvature vector, Bull. Inst. Math. Acad. Sinica, 20 (1992), 53-65.

[97] I. Dimitrić, Quadric representation of a submanifold and spectral geometry, Proc. Symp. Pure Math. 54, Part 3 (1993), 155-168.

[98] I. Dimitrić, Quadric representation of a submanifold, Proc. Amer. Math. Soc., 114 (1992), 201-210 .

[99] I. Dimitrić, 1-type submanifolds of non-Euclidean complex space forms, Bull. Belg. Math. Soc. Simon Stevin, 4 (1997), 673-684.

[100] I. Dimitrić, CR-submanifolds of $H P^{m}$ and hypersurfaces of the Cayley plane whose Chen-type is 1, Kyungpook Math. J., 40 (2000), 407-429.

[101] I. Dimitrić, Low-type submanifolds of real space forms via the immersions by projector, Differential Geom. Appl., 27 (2009), 507-526.

[102] I. Dimitrić, Hopf hypersurfaces of low type in non-flat complex space forms, Kodai Math. J., 34 (2011), $202-$ 243.

[103] U. Dursun, Null 2-type submanifolds of the Euclidean space $\mathbb{E}^{5}$ with parallel normalized mean curvature vector, Kodai Math. J., 28 (2005), 191-198.

[104] U. Dursun, Null 2-type submanifolds of the Euclidean space $\mathbb{E}^{5}$ with non-parallel mean curvature vector, J. Geom., 86 (2006), 73-80.

[105] U. Dursun, On null 2-type submanifolds of Euclidean spaces, Int. Electron. J. Geom., 2 (2009), 20-26.

[106] J. Eells and L. Lemaire, A report on harmonic maps, Bull. London Math. Soc., 10 (1978), 1-68.

[107] J. Eells and L. Lemaire, Another report on harmonic maps, Bull. London Math. Soc. 20 (1988), 385-524.

[108] A. Ferrandez, O. J. Garay and P. Lucas, Finite type ruled manifolds shaped on spherrical submanifolds, Arch fur Math., 57 (1991), 97-104.

[109] A. Ferrandez, O. J. Garay and P. Lucas, On a certain class of conformally flat Euclidean hypersurfaces, Global differential geometry and global analysis (Berlin, 1990), 48-54, Lecture Notes in Math., 1481, Springer, Berlin, 1991.

[110] A. Ferrandez and P. Lucas, P., Null finite type hypersurfaces in space forms, Kodai Math. J., 14 (1991), 406-419.

[111] D. Fetcu, E. Loubeau, S. Montaldo and C. Oniciuc, Biharmonic submanifolds of $C P^{n}$, Math. Z., 266(2010), 505-531.

[112] D. Fetcu, and C. Oniciuc, Explicit formulas for biharmonic submanifolds in Sasakian space forms, Pacific J. Math., 240 (2009), 85-107.

[113] Y, Fu, Biharmonic and quasi-biharmonic slant surfaces in Lorentzian complex space forms, Abstr. Appl. Anal. 2013, Art. ID 412709, 7 pp.

[114] Y, Fu, Biharmonic submanifolds with parallel mean curvature vector in pseudo-euclidean spaces, Math. Phys. Anal. Geom., 16 (2013), 331-344.

[115] Y, Fu, Biharmonic hypersurfaces with three distinct principal curvatures in Euclidean 5-space, J. Geom. Phys., 75 (2014), 113-119.

[116] O. J. Garay, Spherical Chen surfaces which are mass-symmetric and of 2-type, J. Geometry, 33 (1988), 39-52.

[117] O. J. Garay, Finite type cones shaped on spherical submanifolds, Proc. Amer. Math. Soc., 104 (1988), 868-870.

[118] O. J. Garay, An extension of Takahashi's theorem, Geom. Dedicata, 34 (1990), 105-112.

[119] T. Hasanis and T. Vlachos, A local classification of 2-type surfaces in $S^{3}$, Proc. Amer. Math. Soc., 122 (1991), 533-538. 
[120] T. Hasanis and T. Vlachos, Spherical2-type hypersurfaces, J. Geometry, 40 (1991), 82-94.

[121] T. Hasanis and T. Vlachos, Hypersurfaces of $E^{n+1}$ satisfying $\Delta x=A x+B$, J. Austral. Math. Soc. A., 53 (1992), 377-384.

[122] T. Hasanis and T. Vlachos, Surfaces of finite type with constant mean curvature, Kodai Math. J., 16 (1993), 244-352.

[123] T. Hasanis and T. Vlachos, Hypersurfaces in $\mathbb{E}^{4}$ with harmonic mean curvature vector field, Math. Nachr., 172 (1995), 145-169.

[124] T. Hasanis and T. Vlachos, Hypersurfaces with constant scalar curvature and constant mean curvature, Ann. Global Anal. Geom., 13 (1995), 69-77.

[125] C. S. Houh, Rotation surfaces of finite type, Algebras Groups Geom., 7 (1990), 199-209.

[126] C. S. Houh and S. J. Li, Generalized Chen submanifolds, J. Geometry, 48 (1993), 144-156.

[127] S. Ishikawa, Classification problems of finite type submanifolds and biharmonic submanifolds, Doctoral Thesis, Kyushu University, 1992.

[128] C. Jang, Some linearly independent immersions into their adjoint hyperquadrics, J. Korean Math. Soc., 33 (1996), 169-181.

[129] G. Y. Jiang, 2-harmonic isometric immersions between Riemannian manifolds, Chinese Ann. Math. Ser. A, 7 (1986), 130-144.

[130] S. J. Li, Null 2-type surfaces in Em with parallel normalized mean curvature vector, Math. J. Toyama Univ., 17 (1994), 23-30.

[131] S. J. Li, Null 2-type Chen surfaces, Glasgow Math. J., 37 (1995), 233-242.

[132] T. Liang and Y.-L. Ou, Biharmonic hypersurfaces in a conformally flat space, Results Math., 64 (2013), 91104.

[133] Y. Luo, Weakly convex biharmonic hypersurfaces in Euclidean spaces are minimal, arXiv: 1305.71981v1, 2013.

[134] Y. Luo, On biharmonic submanifolds in non-positively curved manifolds and $\epsilon$-superbiharmonic submanifolds, airXiv:1306.6069v1, 2013.

[135] Y. Luo, On the Willmore energy and Chen's conjecture, preprint, 2013.

[136] M. Kotani, A decomposition theorem of 2-type immersions, Nagoya Math. J., 118 (1990), 55-66.

[137] S. Maeta, $k$-harmonic maps into a Riemannian manifold with constant sectional curvature, Proc. Amer. Math. Soc., 140 (2012), 1635-1847.

[138] S. Maeta, Biminimal properly immersed submanifolds in the Euclidean spaces, J. Geom. Phys., 62 (2012), 2288-2293.

[139] S. Maeta, Biminimal properly immersed submanifolds in complete Riemannian manifold of non-positive curvature, airXiv:1208.0473v2, 2012.

[140] S. Maeta, Biharmonic maps from a complete Riemannian manifold into a non-positively curved manifold, ArXiv:1305.7065v1, 2013.

[141] S. Maeta and H. Urakawa, Biharmonic Lagrangian submanifolds in Kähler manifolds, Glasgow Math. J., 55 (2013), 465-480.

[142] A. Martinez and A. Ros, On real hypersurfaces of finite type of CP ${ }^{m}$, Kodai Math. J., 7 (1984), 304-316.

[143] Y. Miyata, 2-type surfaces of constant curvature in $S^{n}$, Tokyo J. Math., 11 (1988), 157-204.

[144] S. Montaldo and A. Ratto, Biharmonic submanifolds into quadrics, arXiv:1309.0631v1, 2013.

[145] S. Montaldo and A. Ratto, Biharmonic submanifolds into ellipsoids, arXiv:1309.1601v1, 2013.

[146] Y. Nagatomo, Finite type hypersurfaces of a sphere, Tokyo J. Math., 14 (1991), 85-92.

[147] N. Nakauchi and H. Urakawa, Biharmonic hypersurfaces in a Riemannian manifold with non-positive Ricci curvature, Ann. Glob. Anal. Geom., 40 (2011), 125-131.

[148] N. Nakauchi and H. Urakawa, Biharmonic submanifolds in a Riemannian manifold with non-positive curvature, Results Math. 63 (2013), 467-471.

[149] N. Nakauchi, H. Urakawa and S. Gudmundsson, Biharmonic maps into a Riemannian manifold of nonpositive curvature, Geom. Dedicata, DOI 10.1007/s10711-013-9854-1. 
[150] Y.-L. Ou, On conformal biharmonic immersions, Ann. Global Anal. Geom., 36 (2009), 133-142.

[151] Y.-L. Ou, Biharmonic hypersurfaces in Riemannian manifolds, Pacific J. Math., 248 (2010) $217-232$.

[152] Y.-L. Ou, Some constructions of biharmonic maps and Chen's conjecture on biharmonic hypersurfaces, J. Geom. Phys., 62 (2012), 751-762.

[153] Y.-L. Ou and L. Tang, The generalized Chen's conjecture on biharmonic submanifolds is false, Michigan Math. J., 61 (2012), 531-542.

[154] B. Rouxel, Chen submanifolds, Geometry and Topology of Submanifolds, VI, 185-198, 1994.

[155] A. Ros, Spectral geometry of CR-minimal submanifolds in the complex projective space, Kodai Math. J., 6 (1983), 88-99.

[156] A. Ros, On spectral geometry of Kaehler submanifolds, J. Math. Soc. Japan, 36 (1984), 433-448.

[157] A. Ros, Curvature pinching and eigenvalue rigidity for minimal submanifolds, Math. Z., 191 (1986), 537-548.

[158] T. Sasahara, Stability of biharmonic Legendrian submanifolds in Sasakian space forms, Canad. Math. Bull., 51 (2008), 448-459.

[159] T. Sasahara, A class of biminimal Legendrian submanifolds in Sasaki space forms, Math. Nachr., Doi 10.1002/mana.201200153, 2013.

[160] T. Tahakashi, Minimal immersions of Riemannian manifolds, J. Math. Soc. Japan, 18 (1966), 380-385.

[161] S. Udagawa, Bi-order real hypersurfaces in complex projective space, Kodai Math. J., 10 (1987), 182-196.

[162] H. Urakawa, Sasaki manifolds, Kähler cone manifolds and biharmonic submanifolds, arXiv:1306.6123v2, 2013.

[163] L. Verstraelen, On submanifolds offinite Chen type and of restricted type, Results in Math. 20 (1991), $744-755$.

[164] L. Verstraelen, Curves and surfaces offinite Chen type, Geometry and Topology of Submanifolds III, 304-314, 1991.

[165] X. F. Wang and L. Wu, Proper biharmonic submanifolds in a sphere, Acta Math. Sin. (Engl. Ser.), 28 (2012), 205-218.

[166] Z.-P. Wang, Y.-L. Ou and H.-C. Yang, Biharmonic maps from 2-sphere, arXiv: 1310.0562v2, 2013.

[167] G. Wheeler, Chen's conjecture and $\epsilon$-superbiharmonic submanifolds of Riemannian manifolds, Intern. J. Math., 24 (2013), no. 4, 1350028 (6 pages).

Department of Mathematics, Michigan State University, East Lansing, Michigan 48824-1027, U.S.A.

E-mail: bychen@math.msu.edu 